\title{
Asesoría pedagógica y formación docente en las universidades argentinas: ¿estrategias apropiadas para un escenario de transformaciones? Aportes desde la investigación
}

Educational \& Pedagogical Advice and teacher training in argentines universities: appropriated strategies for a scenario of transformations? Contributions from research

\author{
Elisa Lucarelli \\ https://orcid.org/0000-0001-7661-8550 \\ elucarel@gmail.com \\ Universidad de Buenos Aires | Universidad Nacional de Tres de Febrero | Argentina
}

\section{RESUMEN}

Desde más de tres décadas el equipo de investigadores del Programa Estudios sobre el aula universitaria focaliza su interés en el análisis de las estrategias orientadas al mejoramiento de la enseñanza universitaria. Desde este supuesto, a lo largo de ese tiempo, encaramos los dos ejes centrales alrededor de los cuales desarrollamos nuestras tareas de investigación. Un primer eje está centrado en las prácticas innovadoras que desarrolla el docente en la enseñanza. Este artículo se referirá al otro eje que se centra en dos de las estrategias que desarrollan las instituciones universitarias: las asesorías pedagógicas y los programas estructurados de formación pedagógica de los docentes acerca de la articulación de ambas estrategias en referencia al lugar que ocupan los cientistas de la educación dentro de los procesos institucionales orientados a la búsqueda de una calidad educativa pertinente.

\section{ABSTRACT}

More than three decades ago, the team of researchers from the University Classroom Studies Program focuses its interest in the analysis of strategies aimed at the improvement of university education. From this course, throughout that time, we face the two central themes around which we develop our research tasks. A first axis is focused on innovative practices developed by the teacher in teaching. This article will refer to the other axis which focuses on two of the strategies that develop the University institutions: The Pedagogical

PALABRAS CLAVE trayectorias científicas, aspiraciones profesionales, experiencia formativa
KEY WORDS

Advisory Teaching, Training Teacher, Practical, Profession Advisory and structured programs of teachers' pedagogical training. We propose in particular considerations about the articulation of both strategies in reference to the place that scientists for education occupy within institutional processes oriented to the search for a relevant educational quality. 
Desde más de tres décadas el equipo de investigadores del Programa Estudios sobre el aula universitariai focaliza su interés en el análisis de las estrategias orientadas al mejoramiento de la enseñanza universitaria. Partimos de la convicción, que estos procesos, desde una perspectiva situada, son posibles y eficientes en función de una convergencia de estrategias macroinstitucionales definidas por políticas centrales (del Rectorado, de la Unidad Académica) y de iniciativas, muchas veces con alto grado de espontaneidad y creatividad que desarrollan los núcleos docentes en su actividad cotidiana para orientar el aprendizaje de los estudiantes.

Desde este supuesto, a lo largo de ese tiempo, encaramos los dos ejes centrales alrededor de los cuales desarrollamos nuestras tareas de investigación. Un primer eje está centrado en las prácticas que desarrolla el docente, que inquietado por los problemas que encuentra en los procesos del aula (y en especial en el aprendizaje de los estudiantes), los enfrenta acudiendo a la búsqueda de formas de enseñanza, que superen las habituales y convencionales que llevaba a cabo hasta entonces. A estas nuevas prácticas formas, superadoras de la rutina en la enseñanza, las reconocemos como innovadoras y acerca de ellas desarrollamos una línea de investigación que se mantiene en la actualidad. Nos interesó y nos interesa conocer de qué manera estos docentes "inquietos" encaran la incorporación de la actividad de los estudiantes en las clases y en la extensión de estas en sus períodos de estudio fuera de esos espacios, desarrollando metodologías, formas de organización del tiempo, de actualización y estructuración del contenido, con inclusión de sus fundamentos epistemológicos, del contexto sociohistórico y de la profesión y de las formas de articulación teoría-práctica.

El otro eje de nuestras investigaciones, al que me referiré en este artículo, se centra en dos de las estrategias (a las que consideramos altamente relacionadas entre sí) que desarrollan las instituciones universitarias: las asesorías pedagógicas y los programas estructurados de formación pedagógica de los docentes. Planteamos en especial algunas consideraciones acerca de la articulación de ambas estrategias en referencia al lugar que ocupan los cientistas de la educación dentro de los procesos institucionales orientados a la búsqueda de una calidad educativa pertinente. 
En primer lugar, nos importa considerar que el Asesor Pedagógico Universitario (APU) puede ser objeto de diversas miradas desde el campo de la Pedagogía y Didáctica Universitarias. En este artículo nos referiremos a la que, analizando sus acciones, lo reconoce como uno de sus actores principales, junto al investigador sobre el campo y al docente disciplinar reflexivo sobre la enseñanza. En efecto, a través de sus prácticas de asesoramiento, formación docente, investigación, intervención, colaboración con la gestión, desarrollo de proyectos institucionales, y de la reflexión sistemática sobre esas acciones, contribuye a la construcción y consolidación de ese espacio disciplinar profesional y lo hace visible en el contexto de la institución. Dentro de este encuadre se lo concibe al APU como sujeto que encarna una profesión de ayuda situada y definida por su contexto histórico social, y que se hace eco de determinaciones institucionales que marcan las características idiosincrásicas de sus prácticas.

Esta perspectiva se hizo presente en las investigaciones que, sobre el asesor pedagógico en las universidades nacionales argentinas, fueron coordinadas por el equipo de Estudios sobre el aula universitaria a lo largo de más de dos décadas. La primera investigación, desarrollada en la década del 90 del siglo pasado, Paradojas de un rol: el asesor pedagógico en la universidad, fue delineada sobre la base de una acción de formación acerca de este rol, y se realizó con la participación de estos actores. Centrada principalmente en el análisis del rol del APU desde su propia perspectiva y desde la de los otros actores de la institución, el núcleo de actividades alrededor de la formación pedagógica de los docentes resulta significativo dentro del conjunto que identificaba su accionar

Así manifestaban los cursantes cuando se les preguntaba acerca de estas (Lucarelli, 2000: 91)

Una de las actividades primordiales [de un APU] es la organización y desarrollo de propuestas destinadas a la formación de sus docentes...

La formación del personal docente se canaliza a través de diversas vías...

También al indagar sobre su percepción de cómo identificaban sus tareas los otros actores institucionales, se hace presente la formación pedagógica como núcleo significativo concretado en diversos formatos:

La demanda institucional apunta a la realización de cursos, talleres o actividades destinadas a la formación de los docentes, ya que de esta forma se pretende mejorar la oferta educativa...

[se nos pide] crear espacios de reflexión para trabajar en el mejoramiento del desempeño pedagógico... 
El análisis de estos testimonios me permite a la vez asomarme a las características estratégicas de esa primera investigación que fue delineada sobre la base de una acción de formación acerca de este rol, con la participación de estos actores. Se trató de un seminario de posgradoii que permitió la realización del estudio citado, el cual se desarrolló metodológicamente con instancias propias de la investigación participativa, dentro de un encuadre de generación conceptual (Rigal y Sirvent, 2012), usualmente denominado cualitativo. Esas instancias permitieron compartir con quienes asistían al Seminario los avances de las reflexiones sistemáticas sobre el problema central acerca de la identidad del APU y sus acciones; a la vez abrieron el espacio para la realización de un meta-análisis acerca del propio seminario por parte de un grupo de participantes, quienes así se expresaron (Lucarelli, 2000: 46),

Durante el seminario se llevó a cabo en forma permanente el análisis de nuestra propia práctica; es decir, situaciones cotidianas de desempeño profesional eran evocadas para ser objeto de reflexiones conjuntas...

En el seminario, el dispositivo pedagógico creado para su dictado favoreció un trayecto de formación centrado en el análisis, ya que brindó conocimientos, métodos y técnicas; permitió la vivencia de diferentes experiencias en el campo de la asesoría...

Nos atrevemos a presentar estas consideraciones con un doble sentido: como antecedente de indagaciones sobre la identidad del APU y a la vez como caso de un modelo de formación alternativo a los cursos convencionales sobre roles institucionales todavía vigentes en las universidades. Y nos preguntamos si la experiencia así vivida por los participantes se podría haber constituido en un elemento referencial para el desarrollo de las acciones de formación pedagógica de los docentes.

A comienzos de la segunda década del presente siglo nuestro equipo de Estudios sobre el aula universitaria encaró otra investigación con foco en el asesoramiento pedagógico en la Universidad.

En el transcurso de los quince años que distancian a esta de aquel primer trabajo, las inquietudes de los APUs por el reconocimiento y la consolidación de su rol en las instituciones dieron lugar a distintas iniciativas tendientes a la visibilización de este actor institucional. Una, de importancia significativa fue la creación de la Red de Asesores Pedagógicos Universitarios (RedAPU) en el 2008, por iniciativa del equipo de la Facultad de Bioquímica y Farmacia de la Universidad Nacional de Rosarioiii. Otra iniciativa fue la realización de eventos académicos para abordar la problemática ligada a ese rol. Precedido por un encuentro metropolitano organizado por la UBAiv , el equipo Coordinador de la RedAPU llevó a cabo -en Rosario- en junio de 2008, el 1 er. Encuentro Nacional de Prácticas de Asesorías Pedagógicas. Estos Encuentros periódicos, cuya sede fue rotativa se realizaron cada dos o tres años en diversas Universidades Nacionales: el segundo en la UBA, 
el tercero, en la UN de La Matanza; el cuarto en la UN de Tucumán, considerado asimismo como el I Latinoamericano, y el V y II Latinoamericano en la UN de Río Cuarto. En términos generales los Encuentros se orientaron hacia profundizar en los abordajes de la asesoría pedagógica universitaria, focalizar en el análisis de las tareas de formación de docentes universitarios, la intervención y la investigación, elaborar estrategias conjuntas para el enfoque de problemáticas comunes a las APUs, reflexionar sobre el impacto de la tarea del APU en las instituciones, fortalecer la RedAPU, y generar espacios de discusión para la construcción y fortalecimiento de la pedagogía universitaria.(Lucarelli y Finkelstein, 2012). Como puede advertirse la formación de docentes universitarios es considerada

La segunda investigación mencionada más arriba se realizó dentro de los alcances de un proyecto argentino conjunto con países del Mercosurv. Cada uno de estos equipos enfocó una faceta particular de un mismo objeto de estudio: el asesor pedagógico universitario comprendido en su contexto, desde un enfoque dinámico que permitiera conocer las condiciones institucionales en que se dan los procesos de conformación del rol, las características que asumen sus prácticas y las relaciones que establece con los otros actores institucionales. Cada uno de estos equipos tomó a la vez caminos diversos en la definición estratégica de un encuadre metodológico común, el de la investigación de generación conceptual (también denominada cualitativa) donde la voz de los sujetos tiene un lugar principal y donde la comprensión de los procesos se asume como prioritaria (Lucarelli, 2015).

Dentro de ese conjunto me referiré a algunos alcances del estudio realizado por el equipo UBAvi , interesado por la identificación de un panorama general de las asesorías pedagógicas argentinas en un conjunto de instituciones nacionales, caracterizando sus problemáticas y actividades según la visión que los propios sujetos tienen sobre su desempeño del rol. El estudio se organizó en dos etapas; la primera, utilizando fuentes secundarias, se desarrolló sobre información empírica contenida en veinte comunicaciones de autoría de esos actores elaboradas el Segundo Encuentro Nacional de Prácticas de Asesorías Pedagógicas. A través del análisis de ese material se elaboró una descripción general sobre el contexto institucional, la estructura organizativa e infraestructura, para analizar consecuentemente las problemáticas centrales que hacen al rol, definidas por núcleos de actividades. En una segunda etapa, se seleccionaron cinco equipos de los explorados anteriormente, considerados como casos, de manera de profundizar, con datos obtenidos a través de entrevistas, los núcleos relevantes de desempeño del rol.

La investigación nos permitió sostener que una mirada amplia desde la complejidad sobre las prácticas del asesor pedagógico universitario trae como desafío interrogarnos cuál es el rol que caracteriza sus prácticas, qué papel cumple dentro de su contexto institucional, de qué manera sus acciones se insertan en el proyecto institucional, y como trasfondo, obligarnos a reflexionar acerca de qué es enseñar hoy en la universidad. 
La investigación nos permitió sostener que una mirada amplia desde la complejidad sobre las prácticas del asesor pedagógico universitario trae como desafío interrogarnos cuál es el rol que caracteriza sus prácticas, qué papel cumple dentro de su contexto institucional, de qué manera sus acciones se insertan en el proyecto institucional, y como trasfondo, obligarnos a reflexionar acerca de qué es enseñar hoy en la universidad.

Entender, en primer lugar, a este actor desde su extranjeridad, (Lucarelli, Finkelstein y Solberg, 2015) supone focalizar el proceso de inserción del APU como pedagogo (o profesionales de campos afines) en las instituciones cuyo eje disciplinar corresponde a otro campo académico o tribu (Becher, 2001). La marca de origen que representa su profesión es un elemento definitorio de la identidad del APU, manifestándose como un condicionante lo suficientemente fuerte para facilitar u obstaculizar su aceptación plena como miembro de la comunidad académica donde desarrolla su labor. Esta tensión no siempre es reconocida explícitamente por el APU, pudiéndose esto interpretarse como una visión más relacionada con el imaginario del deber ser del vínculo con la institución. La extranjeridad implica para este actor reconocer un doble juego: percibirse él mismo como diferente de los otros, y a la vez aceptar que la extranjeridad está presente en los otros, los "dueños del territorio", en cada situación de interacción. Una condición para que esta situación se concrete satisfactoriamente es que los otros, individuales o institucionales, que estén en estado de disponibilidad (Fernández, 2000), esto es, estar libremente disponible para escuchar y aceptar el asesoramiento o identificar la necesidad de esa ayuda por la presencia de una situación institucional crítica.

En estas condiciones y desde reconocerse en esa extranjeridad, el APU en los casos analizados en la investigación desarrolla tareas de asesoramiento directo a docentes, colabora con diversos proyectos institucionales (muchos de carácter administrativos y centrados en el acompañamiento a las trayectorias estudiantiles), y encara acciones de formación pedagógica de los actores "locales" como un modo de promover mejoras en las prácticas de enseñanza; en este último aspecto su desempeño se da a través de distintas modalidades: desde acciones cotidianas hasta el diseño de complejos programas de formación, como pueden ser las Carreras Docentes o los Programas de posgrado; estas actividades involucran diferentes grados de innovación en el statu quo institucional, constituyéndose en algunos casos en verdaderos espacios instituyentes. (Lucarelli, Finkelstein y Solberg, 2015)

En cuanto a la investigación, como campo de prácticas del APU, se observa que si bien esta función es altamente valorada en el contexto institucional en cuanto al área disciplinar, no lo es necesariamente cuando se aplican a lo pedagógico; por su parte el mismo APU, que la reconoce como valiosa, dentro del conjunto de actividades que ma- 
nifiesta realizar, solo un $14 \%$ pertenecen a ese rubro Pareciera que la urgencia de lo cotidiano y de las demandas inmediatas tiene prioridad en la definición del desempeño de este actor, reduciendo la posibilidad de aportes que hacen a la conformación del campo de la Pedagogía y la Didáctica Universitarias.

Pero además de saber a qué se dedican prioritariamente, nos interesó saber quiénes son estos APUs, cuándo fueron creadas las Unidades, con qué nombre se los reconoce, y cuál es su ubicación en la estructura administrativa, el grado de institucionalización del rol. Algunos datos derivados del análisis de esas 20 producciones escritas por los equipos pedagógicos de las universidades nacionales, posibilitan conformar un marco esclarecedor de ese accionar.

La mayoría de los APUs integran equipos, ya sean estos interdisciplinarios o formados solamente por pedagogos; solamente dos de las comunicaciones que mencionan el dato, son unipersonales, también pedagogos.

Si se considera la época de su creación, las producciones escritas muestran que la mayoría de las Unidades son hijas del período democrático iniciado en 1983, ya que sólo dos (de las diecinueve que mencionan el dato) fueron creadas en una época previa. Las otras siete surgieron entre 1993 y diez después del 2001.

La identificación de la Unidad de Asesoramiento Pedagógico a través del nombre con que se las reconoce ya evidencia información de interés; al respecto sostenemos que la diversidad observada en sus denominaciones podría estar acentuando una baja estructuración de este campo profesional. Estas son las denominaciones aportadas: Asesoría pedagógica, Gabinete de Asesoramiento pedagógico, Área de Asesoría pedagógica y Orientación Vocacional, Programa de Orientación, Unidad de Apoyo Técnico a la gestión académica, Instituto Coordinador de Programas de Capacitación Docente, División de Pedagogía Universitaria, Dirección de Pedagogía Universitaria, Servicio de Pedagogía Universitaria, Unidad Pedagógica, Centro Universitario Técnico Educativo. En efecto la mayoría de los que mencionan este aspecto (nueve de quince Unidades que respondieron este ítem) destacan la función que cumplen, mientras otras resaltan el campo disciplinar profesional de referencia. Dentro del primer grupo cinco se reconocen en la asesoría, y los cuatro restantes hacen referencia al apoyo a la gestión, a la formación docente, a la orientación al estudiante, y a esta articulada con la asesoría. Las otras seis que refieren al área disciplinar, cuatro se identifican explícitamente con la pedagogía universitaria y dos solamente mencionan la calificación de pedagógica o técnica al nombrar la unidad de que se trata. Por otro lado, también es dato de interés para comprender la labilidad del campo profesional, el tipo de unidad que define a las Asesorías Pedagógicas dentro de la estructura administrativa, y que se evidencia un amplio espectro de ubicación y jerarquía: División (equivalente a Departamento en la organización por Facultades), Dirección, Instituto, Centro, Gabinete, Área, Unidad, Programa. 
Por otro lado, la mayoría de las Comunicaciones que mencionan el dato, indican que dependen administrativamente de una Facultad (o su equivalente, el Departamento, en aquellas Universidades cuya estructura es Departamental) y una sola señala que se ubica en el Rectorado. En el conjunto que se ubica en las Facultades, la dependencia es de la Secretaría Académica, a excepción de dos que pertenecen una a la Secretaría de Extensión y otra a una cátedra.

El grado de institucionalización de la UAP puede estimarse, por un lado, en función de la normativa que los crea y que establece sus funciones, y a la vez, por la legitimación del rol en la estructura universitaria mediante concursos, ya que estos son los que otorgan ciudadanía a los actores posibilitándoles participar en el gobierno universitario. Hay un número muy alto de los que hacen mención de este aspecto que hablan de la normativa de creación (trece de dieciséis), pero solamente tres se refieren a cargos concursados lo cual permite inferir la existencia de un rol caracterizado por la fragilidad en términos de estabilidad, permanencia y lugar institucional.

Esta rápida semblanza del asesor pedagógico en las universidades nacionales argentinas da marco para hacer foco en una de las líneas principales que identifican sus prácticas como es la formación pedagógica de los docentes.

En la perspectiva institucional el desarrollo profesional se entiende como un conjunto de acciones sistemáticas que se orientan a alterar la práctica, las creencias y los conocimientos profesionales de los docentes, por lo que van más allá de lo informativo [...] En la perspectiva personal, el desarrollo profesional se proyecta por una disposición interna y una postura de búsqueda permanente de crecimiento personal y profesional, disposición de reflexionar sobre las prácticas, actitudes y creencias individuales y colectivas, apertura al cambio (Soares y Da Cunha, 2010: 35. En portugués en el original; la traducción es nuestra).

En el análisis de esta temática la investigación se enmarcó en los aportes realizados por la escuela francesa que abordó la formación como un proceso de construcción personal. En este sentido Gilles Ferry sostiene que la formación implica conformar el propio camino de desarrollo profesional e integral, una trayectoria personal, a través de la búsqueda en sí mismo y en los otros. Afirma que formarse "...es objetivarse y subjetivarse en un movimiento dialéctico que va siempre más allá, más lejos" (1997: 13). Este proceso implica una dinámica en el que intervienen diversas mediaciones: los formadores, las lecturas, las circunstancias de vida, la relación con los otros. Según este encuadre las propuestas institucionales pueden colaborar a que otro se desarrolle, pero no puede sustituir la acción personal intencional para encarar y llevar a cabo la tarea formado. 
Desde la misma perspectiva a la comprensión de los desarrollos formativos, Jean Claude Filloux, permite una profundización metodológica del proceso, a través de la comprensión del enfoque clínico de la formación y al énfasis atribuido a la singularidad que asume ese proceso. Según Filloux el enfoque clínico (centrado en la idea de la relación particular entre sujetos) se sostiene sobre dos ejes: la singularidad del comportamiento de los sujetos, y la posibilidad de generar teoría a partir del conocimiento y la comprensión de lo que a ellos les sucede. (Filloux, 1996).

En el terreno de la formación las acciones fundamentadas en este enfoque se caracterizan por crear situaciones en que las que el formador incorpora explícitamente el conocimiento de sí mismo, de lo afectivo, de lo relacional, como estrategia y contenido de esos programas. En estas definiciones ambos sujetos, el formador y el sujeto en formación, desarrollan un papel activo en el análisis de sus propias motivaciones hacia la enseñanza, de sus prácticas y de sus experiencias de vida, haciendo de esto el eje central de todo el proceso. El retorno sobre sí mismo como mecanismo para abordar el propio conocimiento abre la posibilidad a otros para realizar un camino análogo.

En esta línea de pensamiento, revalorizadora de los procesos de análisis intersubjetivo y de la singularidad de los sujetos del enseñar y el aprender. Marta Souto habla de la formación docente reflexiva entendiendo a esta como "el desarrollo de capacidades que en la profesionalización se plasman como modo de ejercer la profesión reflexivamente..." (Souto, 2017: 71).

Otra dimensión significativa del marco teórico ideológico en que se basa la investigación en cuanto al análisis de los procesos formativos, está centrada en el reconocimiento del valor de la articulación teoría-práctica, tanto en lo relativo a sus fundamentos epistemológicos, como a los pedagógicos y didácticos. Una consideración dialéctica de lo teórico y lo práctico se hace presente en las instancias de formación que desarrollan los docentes, dado que ambas dimensiones atraviesan cualquier actividad de enseñanza y aprendizaje universitarios, cobrando singular importancia por el lugar que tiene en este contexto el aprendizaje de las prácticas profesionales. (Lucarelli, 2009).

En cuanto a la faz organizativa y al sentido de la formación pedagógica de docentes universitarios en servicio pueden reconocerse diversos tipos de modalidades que van desde aquellos desarrollados al interior de los grupos docentes donde prima el aprendizaje por imitación y socialización al compartir la tarea, hasta otros que adoptan diversos grados de formalización ; entre estos últimos se pueden distinguir algunos menos convencionales y con bajo grado de estructuración como los espacios de análisis de las propias prácticas de enseñanza hasta los más formalizados que culminan con certificaciones, y acreditación de títulos de posgrado.

Ickowicz, en su investigación sobre las cátedras universitarias y la preparación de los profesores para la enseñanza, distingue al respecto 
dos modelos de formación: el artesanal y el escolar. El artesanal se desarrolla a semejanza de los procesos que se daban en los gremios en el medioevo en que formación y producción constituían un todo; en él "...el maestro establece los propósitos de la actividad, verifica y rectifica los procesos, domina todo el ciclo de formación en una correspondencia entre formación y trabajo, entre educación y socialización..." (Ickowicz, 2012: 39). En el modelo formal o escolar "la formación se caracteriza por un recorrido en el que intervienen diversos maestros, unos contenidos y modos de transmitirlos predeterminados en el que se fijan tiempos y espacios separados de los espacios de producción" (op.cit. 39). Analiza los rasgos que diferencian ambos modelos según: condiciones contextuales, objeto de conocimiento, condiciones organizacionales, dinámica de las relaciones y concepciones y representaciones; dentro de este último aspecto es de particular significación para este artículo lo que considera acerca de la formación (op.cit.: 41). En el modelo artesanal el proceso se basa en la experiencia, definiéndose los contenidos en función del aprendizaje del oficio y priorizando el saber hacer al desarrollarse en las situaciones en las que se producen los conocimientos. El modelo denominado escolar se distingue porque la formación se centra en la transmisión implicando programas, años de escolaridad y diplomas, a la vez que se prioriza la demostración de saberes adquiridos.

Al respecto de la enseñanza universitaria podemos agregar que mientras que en el primero es el responsable de cátedra, el maestro, quien conduce la formación de sus docentes en las prácticas del oficio (a la manera de un mentor ), en el segundo otros fuera de ese ámbito pueden orientar las acciones formativas; ubicamos en esta situación al asesor pedagógico que, como se señalaba, anteriormente concreta esas acciones a través diversos dispositivos.

\section{Al respecto de la enseñanza universitaria podemos agregar que mientras que en el primero es el responsable de cáte- dra, el maestro, quien conduce la formación de sus docen- tes en las prácticas del oficio (a la manera de un mentor ), en el segundo otros fuera de ese ámbito pueden orientar las acciones formativas; ubicamos en esta situación al asesor pedagógico que, como se señalaba, anteriormente concre- ta esas acciones a través diversos dispositivos.}

En la investigación mencionada en este artículo los testimonios de los APUs dan cuenta de esas modalidades, así como de los distintos tipos de destinatarios, sean estos docentes en ejercicio (o propiamente dichos), docentes iniciantes o noveles y tutores (Finkelstein, 2012)

En cuanto las modalidades como formas menos estructuradas, dirigidas para los docentes en general, indican: 
-Talleres y espacios de reflexión, que articulan marcos teóricos con análisis de experiencias provenientes de sus prácticas cotidianas:

[creamos] un espacio de reflexión pedagógica y de análisis en torno a las prácticas universitarias de enseñanza y evaluación. (UNER. FI, AAPyOV)

Talleres de reflexión con docentes de los primeros años acerca de las problemáticas propias del enseñar y aprender. (UNL. FCA)

... encontramos que el ámbito del grupo de tutores ofrece un espacio fértil para la reflexión sistemática sobre la experiencia, que podría ayudar a vislumbrar maneras efectivas de colaborar con los alumnos en el aprendizaje de los contenidos específicos de las asignaturas que cursan. Poner en primer plano la situación real en la que se encuentran los alumnos que intentan sostener sus estudios lleva a cuestionar no sólo el modo en que se concibe la acción tutorial sino también las prácticas y las propuestas de enseñanza. (UNLu. DE, DPU)

-Cursos y Programas estructurados, alrededor de temáticas relacionadas con los procesos del aula y su contexto

(...) se implementó el curso de Introducción a la Didáctica de la Enseñanza Superior, destinado a los docentes de la Facultad y la enseñanza de la Ingeniería: Los contenidos y la Evaluación. Éste curso, fue pensado y construido a partir de las categorías surgidas de la investigación. (UBA. Fl, AP)

El Programa de Perfeccionamiento del docente de las carreras de Ingeniería.

...En su modalidad continua, consiste en la ejecución de Cursos, Seminarios, Talleres, Paneles, etc. que serán programados anualmente. En su modalidad coyuntural, en la ejecución de actividades similares, pero cronogramadas con 30 días de anticipación. (UNSJ. FI, CUTE).

-Las Carreras de Posgrado correspondientes al área de Pedagógica y Didáctica Universitarias, como acciones formativas de alto nivel de estructuración formal ya que culminan con el otorgamiento de títulos también pueden incluirse de este grupo, y su programación y coordinación forman parte del campo de práctica del APU. Tal es el caso, p.ej. del Instituto Coordinador de Programas de Capacitación (ICPC) de la Facultad de Filosofía y Letras de la Universidad Nacional de Tucumán, creado en 1984 con el nombre de Centro de Pedagogía y Planeamien- 
to Universitario (entonces dependiente de Rectorado). La institución, escenario de juegos paradojales a lo largo de su errática historia de "tensión irresoluta entre permanencia y disolución" (Villagra, 2015: 137), realizó distintas acciones y programas generales de formación de sus docentes, culminando en 1998 con la creación de la Maestría en Docencia Superior Universitaria, primer posgrado en educación de esa Universidad y del noroeste argentino.

-Las Carreras Docentes, programas de formación pedagógica de la Universidad de Buenos Aires instalados en algunas Unidades Académicas para sus profesores, al que nos referiremos en el próximo apartado.

En cuanto a los destinatarios particulares considerados como quienes están en el comienzo de su desempeño profesional pedagógico, como son los docentes iniciantes y los tutores, los asesores pedagógicos los mencionan como sujetos de interés en sus acciones de formación.

-En cuanto a los docentes iniciantes señalan.

Se realiza la Formación pedagógica de los docentes noveles (...) por medio de un Plan de Fortalecimiento al Sistema de Pasantías.... Entendiendo que esta es en la mayoría de los casos, su instancia inicial a su carrera docente. (UNL. FADyU, AP)

...experiencia innovadora de formación y capacitación de alumnos de Grado que ejercen funciones como auxiliares de los docentes de las asignaturas, "centrada en la formación disciplinar-pedagógica, implicó el trabajo interdisciplinario e integrado, promovió una real articulación teórico-práctica, y favoreció la reflexión sobre la propia práctica docente. (UNR. FCByF, SPU)

-También se refieren a la formación de tutores:

El Proyecto de capacitación de Tutores Pares en el Sistema Institucional de Tutorías centrado en la elaboración, implementación y actualización periódica de los dispositivos de capacitación de los aspirantes a tutores (curso/talleres (UNR. FCByF, SPU)

se conformó un equipo de Tutores Alumnos (...) Una vez elegidos ... comenzó la etapa de su capacitación por parte de los Docentes de Asesoría. (UNR. FCA).

En el desarrollo de todas estas acciones de formación pedagógica de los docentes, el asesor en las Universidades nacionales argentinas va haciéndose visible y a la vez consolidando, con distinta intensidad, su espacio y el reconocimiento del rol por parte de los otros actores de ese contexto institucional. 


\section{El asesor pedagógico de la UBA: modalidades de formación en las Carreras Docentes}

Las Carreras Docentes, como se señaló en el apartado anterior, son un espacio de formación docente de la Universidad de Buenos que se constituyen en una instancia estructurada donde tiene un lugar prioritario el accionar del APUviii .

Ubicadas en cinco facultades (Agronomía, Derecho y Ciencias Sociales, Farmacia y Bioquímica, Odontología y Medicina) se distinguen de otros programas de formación pedagógica por centrar su oferta curricular en las problemáticas de enseñanza específicas que se plantean en cada contexto institucional. Otorgan, a excepción de Derecho y Ciencias Sociales, el título de Docente Autorizado, distinguiendo Medicina un primer nivel de cursada cuya titulación corresponde al de Adscripto. Estas dos últimas Carreras son las de mayor antigüedad, ya que fueron creadas en la década del 60 del siglo pasado, siguiendo lo indicado en el Estatuto Universitario en su interés por promover acciones formativas para los docentesix . Agronomía, si bien tiene algunos antecedentes que se remontan a ese origen, recién concreta su Carrera Docente en las dos últimas décadas de ese siglo; otro tanto sucede en Odontología, mientras que la propia de Farmacia y Bioquímica es la última en crearse en 1995. (Finkelstein, 2017).

A su vez la duración de las Carreras y su oferta curricular son dispares en cada Unidad Académica. En cuanto a la duración de los estudios, esta oscila entre las 472 horas (Medicina) y las 80 (Derecho y Ciencias Sociales); las otras Carreras constan, en orden descendente, de 264 horas en el caso de Farmacia y Bioquímica, 250 para Odontología y 208 para Agronomía.

La disparidad observada se hace presente también en las propuestas curriculares en cuanto a los espacios disciplinares y los énfasis que las sostienen. Las cinco Carreras incluyen en sus programaciones espacios dedicados a las formaciones didáctica, en primer lugar, y pedagógica, mientras que las de Medicina y Farmacia y Bioquímica reservan un espacio para la formación académica, dedicada, respectivamente, a contenidos sociohumanísticos y de conocimientos del área disciplinar profesional de la institución. En cuanto a la formación didáctica todas las CD orientan la enseñanza hacia la conformación de una Didáctica específica de la especialidad que distingue a la Unidad Académica respectiva, mientras que dos de aquellas (la de Agronomía y Derecho y Ciencias Sociales) la explicitan como asignatura ("Didáctica Especial") en el Plan de Estudios. Esta orientación da cuenta de la especificidad de las Carreras Docentes, ya así indicadas en su creación, distinguiéndolas de otras ofertas abiertas de posgrados en docencia universitaria. Esta centración en los contenidos disciplinares profesionales como eje de la propuesta curricular y de su implementación es afirmado explícitamente por los APUs.

En este sentido es esclarecedor para este aspecto lo que afirman estos actores al describir el Plan de estudios y el tratamiento pedagógicos de los contenidos específicos 
Y el módulo 3 es de Didáctica Especial de la Disciplina, sería como de didáctica específica (...), este año tenemos un programa muy centrado sobre las estrategias pero revisando algunos artículos críticos sobre cómo se enseña Derecho y algunos artículos interesantes de epistemología del Derecho. (Entrev. PCD FDyCS).

Yo doy la pedagogía y la didáctica especial (...) que es una didáctica orientada a la enseñanza de las ciencias y tecnologías agropecuarias y ambientales. Y ahí lo hacemos en un equipo en el cual está toda la visión ingenieril (...) (Entrev.DCD FA)

en Planeamiento vamos mirando los contenidos y viendo si están planteados como contenidos conceptuales o procedimentales, si realmente se los voy a enseñar como un proceso... o, por ejemplo, "historia clínica" la voy a enseñar como un concepto o cómo confeccionar la historia clínica y todos los pasos que me requiere la confección de una historia clínica? (Entrev. DCD FO)

Los asesores pedagógicos fueron los principales responsables de la elaboración de los planes de estudios, aprobados luego por Resoluciones del Consejo Directivo de cada Facultad y el Superior de la Universidad.

Aún cuando la dirección formal de las Carreras pueda estar en las Secretarías académicas o de Posgrado de las facultades, los Coordinadores reales del programa son los APUs; ellos son pedagogos, con excepción del caso de Medicina, en que el cargo es ocupado por un médico. La CD de Derecho y Ciencias Sociales está coordinada por quien tiene la formación disciplinar (es abogado) y pedagógica (es también licenciado en Ciencias de la Educación)

Además de ese lugar central en cuanto al planeamiento curricular y la coordinación de su implementación los asesores pedagógicos tienen a su cargo el desarrollo parcial o total de las clases correspondientes a la formación pedagógica y didáctica, tal como lo evidenciaron los testimonios anteriormente citados.

A través de estas funciones centrales que desempeña en las $C D$ como son el diseño del currículum, coordinación de su implementación y el mismo ejercicio de la docencia, consideramos que el APU va configurando una impronta significativa en el estilo pedagógico didáctico de estos programas. Y nos preguntamos si esta conformación no se constituye a la vez en un camino de legitimación (no escrita ni reconocida normativamente) de su rol en la unidad académica. 
Podemos analizar, a través de sus propias expresiones, cómo el APU visualiza el estilo pedagógico didáctico de la CD a través de dos aspectos: la búsqueda de estrategias metodológicas de enseñanza que propicien un rol activo del cursante en su propio aprendizaje y, consecuentemente con esto, la instalación de la práctica como eje de la profesionalización docente.

¿Qué dicen los APU, a través de las entrevistas, acerca de las estrategias metodológicas de enseñanza que se implementan en las CD?

\section{En Odontología}

... nosotros hacemos mucho hincapié en mostrar otras técnicas que tengan la vista en el rol más activo del alumno, entonces hacemos muchas prácticas. Incluso en los módulos trabajan mucho con casos, con problemas, con otras metodologías que tengan que ver con salir de lo tradicional (...) hacen microclases con otras propuestas de enseñanza que sean diferentes a las que están más instaladas en nuestras propias cátedras... que puedan ver qué otras posibilidades de enseñar tienen que por ahí son más potentes para el aprendizaje del alumno. ...en los módulos de Recursos Didácticos se ven específicamente recursos que puedan facilitar la enseñanza de la odontología, entonces se trabaja mucho con simulaciones, con imágenes, con videos...

\section{En Agronomía}

...trabajamos con ateneos (...) en cuanto a los cursos lo que hacemos es trabajar muchísimo con clínicas en donde tomamos casos, además de elementos teóricos por supuesto, lectura... trabajamos bastante, menos de lo que querríamos, con lo que es micro enseñanza... después, por supuesto lecturas de todo lo que haya

\section{En Medicina}

... tratamos de hacer una clase dinámica con resolución de casos, trabajos en pequeños grupos, mucho trabajo práctico. Analizan programas e instrumentos de evaluación, trabajan diferentes habilidades docentes, hay debate y discusión. Usamos la metodología de taller. Tienen muchas dificultades para incorporar la tecnología.

\section{En Farmacia y Bioquímica}

ahora desde la clase les marcamos que tienen que enmarcar un problema o empezar a delimitar un problema, y a lo 
largo de las clases, y a través del desarrollo temático de la materia lo que van haciendo es abordar esos temas, y esos temas van enriqueciendo el problema y van avanzando en posibles soluciones. $Y$ esas soluciones tienen que estar vinculadas con sus prácticas docentes.

\section{En Derecho}

Tienen que indagar sobre alguna estrategia, indagarla en bibliografía, en investigaciones, en eso estamos trabajando ahora, y luego hacer un trabajo que van a presentar en modalidad seminario... hoy les pedí ese resumen y hoy les voy a hacer intercambiarse los resúmenes, dárselo al de al lado y que el compañero le comente el resumen...

Se hacen Microclases pero en la propia aula en la que se dicta el módulo, simulacros de clases, no frente a alumnos reales... [sino] ante los propios compañeros de formación docente.

Estos testimonios evidencian que la enseñanza en las Carreras Docentes, según los APU, se desarrolla a través de un conjunto variado de estrategias: la presentación de problemas; el uso de casos; la utilización de recursos didácticos con simuladores, imágenes, videos; la inclusión de ateneos, talleres, debates y microclases; el trabajo en grupos; la asignación de lecturas bibliográficas y análisis evaluativo de documentos. Y seguramente, aunque no lo mencionan, también la exposición en sus diversos formatos.

\section{la enseñanza en las Carreras Docentes, según los APU, se de- sarrolla a través de un conjunto variado de estrategias: la presentación de problemas; el uso de casos; la utilización de recursos didácticos con simuladores, imágenes, videos; la inclusión de ateneos, talleres, debates y microclases; el trabajo en grupos; la asignación de lecturas bibliográficas y análisis evaluativo de documentos.}

Todas las estrategias mencionadas permiten inferir el desarrollo de clases orientadas a propiciar un proceso genuino de aprendizaje donde el rol activo del cursante docente es el eje de la estructura didáctica. Analizadas desde el tipo de modalidades particulares de articulación teoría-práctica a que esas estrategias dan lugar, podemos advertir que las mencionadas se caracterizan por ser complejas y, a la vez, propiciar una articulación dialéctica entre aspectos teóricos y prácticos; en este sentido "las actividades emprendidas por... [el cursante] son de mayor grado de complejidad y autonomía en la construcción del conocimiento", (Lucarelli, 2009:97), presentando un grado alto de orientación hacia el aprendizaje significativo. Esta primera vía principal de la articulación teoría-práctica, según este enfoque, se complementa con una segunda, 
entendida como "proceso particular de adquisición de conocimientos, actitudes, formas de operar específicas relativas a la práctica profesional" ... (op.cit., 96), en este caso de un docente universitario.

Si bien esta intencionalidad hacia la profesión se desarrolla a lo largo de todo el trayecto formativo, es interesante analizar cómo se incluye en los diseños curriculares el espacio dedicado específicamente a las prácticas de la profesión docente en el ámbito universitario.

Considerar este espacio curricular como analizador privilegiado, da lugar para recordar que las CD son programas de formación continua de docentes en ejercicio, portadores estos de concepciones acerca de que es enseñar y qué es aprender en la universidad. Su práctica cotidiana da cuenta de un conjunto de acciones a través de las cuales expresa (muchas veces sin asumirlo de manera consciente) su marco de referencia teórico valorativo acerca de los procesos pedagógicos y didácticos que se viven en la unidad académica en general y en el aula en particular . Las teorías implícitas (Bruner, 1988) conforman ese marco constituyendo un tejido complejo de conocimientos e intuiciones basados en la historia personal y colectiva a partir de las cuales el profesor organiza su acción educativa y los procesos formativos.

Y precisamente un propósito significativo de la formación pedagógica de los docentes en estas Carreras podría ser el esclarecimiento de los aspectos oscuros, no revelados de ese marco; el espacio curricular de la Práctica Profesional pareciera ser particularmente apropiado para esa toma de conciencia a través, tanto de los distintos mecanismos de organización del Espacios para Práctica Docente en el diseño curricular, como del rol que desempeña el APU como formador en el desarrollo y evaluación de ese espacio.

Tal como se señaló precedentemente cuatro son las CD de la UBA en las que ese espacio está presente con distinto grado de intensidad horaria: Odontología, Agronomía, Farmacia y Bioquímica, y Medicina. En este último caso, el asesor pedagógico no tiene injerencia, ya que la supervisión y evaluación del cursante durante los cinco cuatrimestres en que se cumple este espacio curricular, esas funciones son ejercidas por el profesor a cargo de la cátedra o el responsable del servicio o departamento en que esa práctica se cumple; los informes evaluativos son remitidos por ellos al Director de la Carrera.

En una situación opuesta en cuanto tanto a la participación y responsabilidad del APU, como al significado dado a la enseñanza de las prácticas, se encuentra el caso de Odontología, centrándose estratégicamente en los procesos reflexivos sobre las prácticas cotidianas de un docente a lo largo de todos los módulos de la Carrera. Así lo manifiestan las entrevistas:

siempre la práctica docente es una mirada de reflexión sobre la práctica [más que] instalar una nueva práctica...todos [los cursantes] tienen práctica docente. Entonces ese es un punto de partida que para nosotros es esencial porque ellos realizan su práctica todos los días, entonces un momento 
muy importante es instalarse en la reflexión y ver cómo hacemos esto, qué se hace, qué se piensa, más que [desarrollar otra nueva]... práctica (Directora de CD)

La Práctica docente es un trabajo de análisis de su propia práctica, en alguna dimensión de su propia práctica. Por ejemplo, hubo años en que abordamos el tema de la evaluación. Hicimos una propuesta en donde desarrollaban como un dispositivo de observación de situaciones de evaluación y analizaban esas situaciones de evaluación dentro de sus propias prácticas...ellos tienen que reflexionar sobre sus propias prácticas y ponerles luz teórica a esas prácticas para poder conservarlas, modificarlas, transformarlas. (Docente de CD)

La articulación dialéctica entre práctica y teoría, experiencia y conceptualización, aparece claramente manifiesta en el testimonio, mostrando a la formación como un proceso de cambio de los comportamientos profesionales habituales, revelándose esa instancia curricular como un espacio de innovación. Si bien la práctica hegemoniza la enseñanza en todo el desarrollo del Programa, hay instancias específicas de producción sobre este aspecto en dos momentos del trayecto: al finalizar el primer año y al término del segundo como culminación de la Carrera. Todas estas instancias son orientadas, supervisadas y evaluadas por el equipo APU.

Dentro de un enfoque clínico de la formación, se trata de acciones tendientes a lo que Souto denomina formación docente reflexiva, entendida como

...desarrollo de capacidades que en la profesionalización se plasman como modo de ejercer la profesión reflexivamente; como herramienta a ser incorporada por el docente para su quehacer; como actitud ante la realidad profesional, permitiendo cambios en el modo de vinculación con la realidad, más que en la incorporación de un saber o un conocimiento precisos. (Souto, 2017: 71).

Por su parte, la Práctica Profesional Supervisada (PPS) de la CD de la Facultad de Farmacia y Bioquímica también es eje del trabajo formativo del equipo de APU, realizado a través de procesos de análisis de las prácticas que desarrolla el cursante. Esos procesos, que son llevados a cabo al finalizar cada módulo del Programa, se consolidan en el espacio de la PPS ya que el cursante elige una clase (que será objeto de observación por parte del equipo APU), la programa y realiza el análisis posterior con su docente.

desarrollan la clase con alumnos reales y nosotros vamos a ver la clase y hacemos una devolución sobre esa clase y ellos analizan su propia clase también en un informe final... 
Ellos reconocen avances y eso para nosotros es clave. El hecho de poder transparentar decisiones, saber por qué están decidiendo lo que están decidiendo, porqué tomaron este camino y no otro, porqué deciden encarar la clase desde un lugary no desde otro

Se advierte por parte del equipo APU el desarrollo de un proceso de acompañamiento del cursante en las distintas instancias de la práctica de enseñanza y de su programación, asumiendo un rol tutorial orientador de los procesos reflexivos.

Por último, el Espacio de la Práctica de Enseñanza (que ocupa casi la mitad del total de horas de la $\mathrm{CD}$ ) en la Facultad de Agronomía habla de una acción compartida entre los equipos APU y el equipo de la cátedra de procedencia del cursante, utilizando un recurso específico como es el dossier. El espacio curricular implica el desarrollo de un proceso graduado a lo largo de dos años, centrado en las diversas actividades que constituyen las prácticas de un docente en la universidad, además de las actividades frente a estudiantes. Así se expresa la Directora en la entrevista

lo que quisimos con los profesores era que les habilitaran espacios ... [para] ellos, entre las prácticas, conducir reuniones, conducir consultas de estudiantes...ir a las reuniones de cátedra.

Esta modalidad de trabajo articulado y colaborativo ente el APU y las cátedras se hizo evidente en el dossier, recurso para orientar el análisis de la propia práctica por parte del cursante. El proceso mismo de elaboración del documento orientador, Agropaideia (2013), propuesto por los pedagogos, fue largo y complejo; se lo entiende como

un conjunto de documentos que reúne la producción intelectual de una persona y evidencia la marcha de su proceso de aprendizaje. Es un instrumento de autoevaluación y autoadministración que permite apreciar los propios avances -en este caso de la práctica docente- e incorpora diálogo con el profesor supervisor...así como los de sus colegas (op.cit.:4).

El profesor titular de la cátedra de pertenencia de cada cursante es el supervisor de la práctica y quien evalúa los Informes parciales y final, el cual, firmado por el cursante es remitidos al APU, Director de la Carrera, para su inclusión en el record correspondiente. Durante todo este período el APU sostiene reuniones individuales y colectivas con los cursantes a modo de acompañamiento y orientación de esas prácticas.

Unas palabras finales: en función de todo lo anteriormente expuesto sostenemos, una vez más, que los procesos y estrategias institucionales, como las asesorías pedagógicas y la formación del docente universita- 
rios (así como innovaciones en la enseñanza) sólo pueden ser eficientes si se los comprende en función de los sujetos que intervienen y del contexto situacional histórico al cual pertenecen, es decir, en el sentido que da a eficiencia Vera María Candau (Candau, 1988: 16),

\section{los procesos y estrategias institucionales, como las asesorías pedagógicas y la formación del docente universitarios (así como innovaciones en la enseñanza) sólo pueden ser eficien- tes si se los comprende en función de los sujetos que inter- vienen y del contexto situacional histórico al cual pertenecen}

La búsqueda de la eficiencia no se niega. Muy por el contrario. Pero la propia eficiencia pedagógica es repensada cuando se parte del compromiso con la transformación social que exige prácticas pedagógicas adecuadas a la mayoría de la población del país. 
i Se trata de un Programa de investigación situado en el Instituto de Investigaciones en Ciencias de la Educación de la Facultad de Filosofía y Letras de la Universidad de Buenos Aires, dirigido por Elisa Lucarelli entre 1985 y 2015, y desde entonces a la actualidad por Claudia Finkelstein.

ii "Paradojas de un rol: el asesor pedagógico en la universidad", en el ámbito de la Cátedra de Didáctica de Nivel Superior y del Programa Estudios sobre el aula universitaria, de la Facultad de Filosofía y Letras de la Universidad de Buenos Aires. Coordinados por Elisa Lucarelli y con la participación como docentes de María E. Donato, Isabel Abal de Hevia, Martha Nepomneschi e Irma Zacaría, El curso se repitió en tres cuatrimestres entre los años 1995 y 1996, contando con la participación de asesores pedagógicos de universidades nacionales y privadas del país. Los hallazgos de la investigación correspondiente dieron lugar a un libro (Lucarelli, 2000) publicado con el mismo título; en el mismo también colaboraron con la elaboración de un capítulo dos cursantes del Seminario, Claudia Finkelstein y Claudia Faranda.

iii En la conformación y desarrollo de esta Red tuvo un papel primordial María Alejandra Pardal quien, con su equipo del Servicio Pedagógico de la institución mencionada, sostuvo las acciones preliminares y permitió la difusión a nivel de todo el país. Al respecto señala: "Adhiriendo al paradigma de trabajo en Red, se propuso la constitución de la RedAPU, no como un evento sino como un proceso que se desarrollará a lo largo del tiempo, del que se conocería su inicio, pero no su desenlace"( Pardal, 2014: 184).

iv Se trata del Primer Encuentro de asesores pedagógicos de Universidades Nacionales de la Capital Federal y el Conurbano, organizado por el Programa de Estudios universitarios (IICE, FFyL) y el Área de Educación Odontológica y Asistencia Pedagógica (FO) de la Universidad de Buenos Aires, desarrollado el 28 de marzo de 2008, con el propósito de facilitar el intercambio entre los equipos de Asesores Pedagógicos de las Universidades Nacionales de ese ámbito y promover la circulación de información sobre las experiencias pedagógicas llevadas a cabo en las diferentes Unidades.

V Proyecto Conjunto de Investigación Mercosur Argentina (SPU)-Brasil (CAPES) PPCP 003/11: Estrategias institucionales para el mejoramiento de la calidad de la Educación Superior y el desarrollo profesional docente, que fue coordinado por la Dra. María Isabel Da Cunha por Brasil desde la Universidade Do Valle Do Sinos (UNISINOS) y por mí, desde la Universidad de Buenos Aires. El Proyecto, de dos años de duración, incluía, además de acciones de formación de posgrado en ambos países e intercambio de docentes, un componente de investigación que permitía la inclusión de otros equipos institucionales, además de los propios de las universidades coordinadoras. Así se incorporó Uruguay, con el equipo de la Comisión Sectorial de Enseñanza de la Universidad de la República, y de Argentina, además de la UBA, participaron equipos de la Universidad Nacional de Tucumán y de la Universidad Nacional del Sur.

vi Elisa Lucarelli, Claudia Finkelstein y Viviana Solberg, contando con la colaboración, como adscripta al equipo de investigación, de María Mercedes Lavalletto.

vii Mayor Ruiz y Sánchez Moreno (2000:20-1) así lo definen: "Profesor mentor es un profesor experto que asiste al nuevo profesor, le ayuda, le da orientaciones tanto en el currículum como en la gestión de la clase. Se los caracteriza como expertos, con habilidades de reflexión y análisis de la enseñanza, dispuestos a ejercer esa labor de acompañamiento y responsable en sus tareas de docencia. El profesor mentor actúa como modelo demostrando sus destrezas docentes frente a la clase y en el resto de las actividades"

viii El contenido de este apartado remite a la investigación La formación pedagógica del docente universitario: las Carreras Docentes en la Universidad de Buenos Aires que, bajo la dirección de Claudia Finkelstein, está realizando el equipo del Programa Estudios sobre el aula universitaria (IICE, FFyL, UBA), integrado también por Elisa Lucarelli, Gladys Calvo, Walter Viñas y María Mercedes Lavalletto. Esta investigación que, se encuentra en la elaboración de las Conclusiones finales y en proceso de publicación forma parte del Proyecto NEIES (Núcleos de Estudios e Investigaciones en Educación Superior) del Mercosur: Estrategias institucionales para la formación pedagógica de los docentes de nivel superior orientadas al mejoramiento de la calidad del nivel superior de educación, con Dirección por Argentina de Claudia Finkelstein, en el que participan, además de la UBA, la UNTucumán, la UNSur, por Argentina; la UdelaRepública por Uruguay; y la UFPelotas y la UEBahia por Brasil.

ix Art. 67.- La carrera docente se adapta a la estructura de cada una de las Facultades y puede comprender las tres categorías de auxiliares docentes mencionados en el art. 65 , o bien puede tener un régimen especial para el otorgamiento del título de docente autorizado; implica la asistencia a cursos y seminarios sobre temas vinculados a la respectiva asignatura y la participación en esos cursos y seminarios así como también la asistencia y la participación en cursos de metodología de la enseñanza y la investigación. La Universidad organiza los cursos especiales que las Facultades requieren para el cumplimiento del fin establecido en el presente artículo. (UBA. Estatuto Universitario. 1958).

$\mathrm{x}$ Vale reiterar que, en este contexto, el de la universidad, el aula es tanto el salón de clases, como también el laboratorio, la sala de hospital, el consultorio, el taller, el estudio, la institución de servicio profesional, la empresa o cualquier terreno donde el estudiante pueda desarrollar acciones formativas propias de la profesión de destino. 
APU: Asesor Pedagógico Universitario

CD: Carrera Docente

Entrev. PCD FDyCS: Entrevista Profesor CD Facultad de Derecho y Ciencias Sociales.

Entrev. DCD FA: Entrevista Docente CD Facultad de Agronomía

Entrev. PCD FDyCS: Entrevista Profesor CD Facultad de Derecho y Ciencias Sociales.

Entrev. DCD FO: Entrevista Docente CD Facultad de Odontología

ICPC: Instituto Coordinador de Capacitación Docente.

NEIES: Núcleos de Estudios e Investigaciones en Educación Superior

PPS: Prácticas Profesionales Supervisadas

UBA: Universidad de Buenos Aires.

UNT: Universidad Nacional de Tucumán

UNS: Universidad Nacional del Sur

UdelaR: Universidad de la República

UFPel: Universidade Federal do Pelotas

UEB: Universidade Estadual do Bahia

UNER. FI, APyOV: Universidad Nacional de Entre Ríos. Facultad de Ingeniería. Asesoría Pedagógica y Orientación Vocacional.

UNL. FCA: Universidad de Nacional de Litoral. Facultad de Ciencias Agrarias.

UNLu. DE, DPU: Universidad Nacional de Luján. Departamento de Educación. División de Pedagogía Universitaria. 


\section{BIBLIOGRAFIA}

Bruner, Jerome (1988). “Desarrollo cognitivo y educación”. Madrid: Morata.

Becher, T. (2001). “Tribus y territorios académicos. La indagación intelectual y las culturas de las disciplinas". Barcelona. Gedisa.

Candau, V.M. (1990). “Rumo uma nova didáctica”. Rio. Vozes, 1990.

Carrera Docente. Facultad de Agronomía. Universidad de Buenos Aires. (2013). "Agropaideia. Dossier de la Práctica". Buenos Aires.

Fernández, L. (2000). “Prólogo. En: Lucarelli, E. (comp.): El asesor pedagógico en la universidad: de la teoría pedagógica a la práctica en la formación". Bs.As. Paidós.

Ferry, G. (1997). “Pedagogía de la formación". FFyL,UBA - Novedades Educativas.

Filloux,J.C.:(1996). “Intersubjetividad y formación". Bs As. FFyL,UBA - Novedades educativas.

Finkelstein, C. (2012). "Acerca de las acciones de formación. Proyecto Conjunto de Investigación Mercosur Argentina". (SPU) - Brasil, (CAPES) PPCP 003/11, Estrategias institucionales para el mejoramiento de la calidad de la Educación Superior y el desarrollo profesional docente. Buenos Aires. UBA. FFyL IICE. Documento interno.

-----(2017). "La formación de los docentes universitarios en la UBA: modalidades, dispositivos, formatos. Primer avance de investigación". Proyecto NEIES Estrategias institucionales para la formación pedagógica de los docentes de nivel superior orientadas al mejoramiento de la calidad del nivel superior de educación. Bs. As. UBA. FFyL IICE.NEIES. Documento interno.

Ickowicz, M. (2002). "Los trayectos de formación para la enseñanza en profesores universitarios sin formación docente de grado". Tesis de Maestría. UBA. FFyL. Maestría en Didáctica. Buenos Aires.

Lucarelli,E. (Comp.)( 2000). “El asesor pedagógico en la universidad: de la teoría pedagógica a la práctica en la formación". Bs.As. Paidós.

Lucarelli, E. (2009). “Teoría y práctica en la universidad. La innovación en las aulas". Bs.As. Miño y Dávila.

Lucarelli, E. y Finkelstein, C.(ed.) (2012). “El asesor pedagógico en la universidad. Entre la formación y la intervención". Bs. As. Miño y Dávila.

Lucarelli, E. (ed.) (2015). “Universidad y asesoramiento pedagógico". Bs. As. Miño y Dávila. 
Lucarelli, E. (2015). “Presentación”. En: Lucarelli, E. (ed.) Universidad y asesoramiento pedagógico. Bs. As. Miño y Dávila.

Lucarelli, E., Finkelstein, C. y Solberg, V. (2015). “Una mirada actual del asesor pedagógico universitario: escenarios y trayectos". En: Lucarelli, E. (ed.): Universidad y asesoramiento pedagógico. Bs. As. Miño y Dávila.

Mayor Ruiz, C. y Sánchez Moreno, M. (2000). “El reto de la formación de los docentes universitario. Una experiencia con profesores noveles". Sevilla. U. de Sevilla.ICE.

Pardal, M. A. (2014). “Asesoramiento pedagógico en la universidad. Prácticas, escenarios y protagonistas en una Facultad de Bioquímica y Farmacia". Saarbrucken. Alemania. Editorial Académica Alemana.

Rigal, L. y Sirvent M.T. (2012). "Metodología de la Investigación Social y Educativa: Diferentes caminos de producción de conocimiento". Buenos Aires. Miño y Dávila. (Manuscrito en vías de revisión).

Soares, S.R. y Da Cunha, M.I. (2010). "Formaçao do profesor, a docencia universitaria em busca de legitimidade. Salvador, Bahía. EDUFBA.

Souto, M. (2017). “Los pliegues de la formación. Sentidos y herramientas para la formación docente". Rosario. Homo Sapiens.

UBA. (1958) Estatuto Universitario. 